Article

\title{
Galactic Density and Evolution Based on the Virial Theorem, Energy Minimization, and Conservation of Angular Momentum
}

\author{
Robert E. Criss * and Anne M. Hofmeister \\ Department of Earth and Planetary Science, Washington University, St. Louis, MO 63130, USA; \\ hofmeist@wustl.edu \\ * Correspondence: criss@wustl.edu; Tel.: +1-314-935-7441
}

Received: 25 May 2018; Accepted: 9 November 2018; Published: 15 November 2018

check for updates

\begin{abstract}
Spiral galaxies are spinning, internally densified objects. The Virial Theorem explains galactic rotation curves via its linkage of the rotation rate to the gravitational self-potential $\left(U_{g}\right)$ and the moment of inertia of oblate spheroids. We devise a new analytical solution that allows galactic mass and volumetric density $\left(\mathrm{kg} \mathrm{m}^{-3}\right)$ profiles to be extracted from velocity and its derivative as functions of equatorial radius. This inverse model of rotation curves is direct, unambiguous, and parameter-free. To probe galactic evolution, we combine energy minimization, angular momentum conservation, and the Virial Theorem. The characteristic flat shape of spiral galaxies results from an initial vertical collapse of a spinning, colossal molecular cloud, which reduces $U_{g}$ while conserving angular momentum. Subsequent inward densification further lowers $U_{g}$, producing bulges, but conserving angular momentum requires mass loss, achieved by the outward movement of the distal parts of the spiral arms. Many of the evolutionary patterns of spiral galaxies are exhibited by the changing shapes of hurricanes during formation and dissipation. In contrast, elliptical galaxies evolve from a cloud with roughly random orbits into progressively rounder, internally denser objects, with angular momentum conserved by the development of vertically oriented jets. Galactic evolution is governed by the initial inventory of mass and angular momentum, resulting in separate paths for elliptical and spiral galaxies, as is codified in Hubble's tuning fork diagram.
\end{abstract}

Keywords: inverse models; rotation curves; evolution; angular momentum; dissipation; spiral galaxies; elliptical galaxies

\section{Introduction}

Subsequent to Hubble's discovery of galaxies, explaining their shape, character, rotation and evolution have been major goals of astronomy and astrophysics. The properties of these objects have been so confounding that explanations at odds with observations and demonstrably correct laws of physics have been offered. For example, Newtonian orbital models of galactic rotation require large amounts of hypothetical, non-baryonic matter (reviewed by [1]), to reproduce the measured dependence of tangential velocities on radius (rotation curves, RC). To avoid calling on the presence of matter that does not interact with light, Milgrom [2] proposed an ad-hoc modification to Newton's law of gravitation. Although most subsequent publications on galactic rotation adhere to one or the other of these incompatible views, several independent research groups with different approaches [3-7] provide explanations of RC that are rooted in Newtonian physics and known forms of matter; see also [8]. Here we further develop our quantitative explanation, specifically that galaxies are spinning, oblate bodies constituted of ordinary matter that are governed by classical physical laws. 
The link between the spin and shape of self-gravitating objects was important long before galaxies were known to exist. Newton was interested in this problem, because an accurate depiction of Earth's shape was essential to map-making and celestial navigation [9]. Newton made the first reasonable estimate of Earth's flattening, but the superior analysis by Maclaurin [10] and Todhunter [9] links the shape and spin of dense, oblate objects that have lost most of their initial inventory of spin energy, e.g., [11,12]. A century later, Clausius [13] developed his Virial theorem to further thermodynamics, but his remarkable relationship applies to any bound, conservative system, including galaxies [14].

The Virial Theorem (VT) quantifies the ratio of the potential to kinetic energies of bound systems, yet was derived without specifically invoking energy or momentum conservation. On a fundamental level, the VT is based on symmetry [14]. For these reasons, the VT provides an additional dynamical constraint on bound systems that can be used along with the well-established principles of energy and momentum conservation. However, galaxies are evolving systems. To treat evolutionary behavior in thermodynamic assessments, energy minimization must be considered, because evolving systems are dissipative [15]. Heat being shed is a primary dissipative process.

The present paper combines the VT with conservation of angular momentum to determine what processes are consistent with energy minimization as galaxies evolve. We show how galactic shape, spin rate, and evolution are directly linked to the gravitational self-potential $\left(U_{g}\right)$. We also develop an inverse model that allows detailed volumetric density variations in galaxies to be directly and simply extracted from their rotation curves. Inverse models differ from forward (fitting) models in fundamental ways, and are of paramount importance to astronomy as discussed by Groetsch [16]. One example is Ambarzumian's [17] method for extracting true velocities from data on radial velocities.

Our paper directly computes volumetric density $\left(\mathrm{kg} \mathrm{m}^{-3}\right)$ with no free parameters. Previous studies (e.g., [18]) use forward models to explore surface density $\left(\mathrm{kg} \mathrm{m}^{-2}\right)$, which is a different physical quantity that requires either a free parameter (e.g., scale length) or other assumptions to approximate volume density (e.g., [19]). Some studies determine surface mass by modelling luminosity, and use this information in multi-component fits of rotation curves (e.g., [20]). Section 2.1 and 2.2 summarize previous forward models.

Finally, we use the hurricane analogy proposed earlier, on the basis of similarities in morphology and velocity profiles [7], to gain further insight into the behavior, evolution, and abundance of spiral galaxies. We explain why spirals differ greatly in shape, size and complexity from elliptical galaxies, and provide a possible evolutionary link to Hubble's famous tuning fork diagram. For a recent review of previous models on galactic evolution, see [21].

\section{Previous Efforts to Analyze Rotation Curves}

Prior to this work, no studies had extracted volumetric density $\left(\mathrm{kg} \mathrm{m}^{-3}\right)$ or mass profiles directly from galactic rotation curves using an inverse procedure, which by definition involves no fitting (e.g., [16]). Although some analyses of RC measurements have been called inverse models, these involve fitting and calculate surface density: see the summary of Marr [4]. Most efforts are forward models that either use central forces or assumed potential functions, to calculate RC from presumed density distributions. These Newtonian Orbital Models (NOMs) commonly include central bulges, galactic discs, black holes, and giant haloes constituted of non-baryonic particles, now considered to be a major component of our universe. Another approach involves ad hoc modifications to Newton's law of gravitation (MOND; after Milgrom [2]). For a review, see McGaugh [22]. Relativistic approaches (e.g., [23]) and MOND are not discussed further because we focus on Newton's confirmed law.

\subsection{Force Models}

Early studies of RC considered centrally directed gravitational forces to calculate orbits of test masses within galaxies. The associated formula for the dynamical mass, derived by equating centripetal force $m v^{2} / r$ on orbiting test particles (mass $m$ ) to Newton's attractive force, remains widely used: 


$$
v^{2}(r)=\frac{G M_{i n}}{r}
$$

where $M_{\text {in }}$ is the total mass interior to a particular orbit (e.g., [24]). Although this equation works well for planetary orbits about a massive central body, which can be represented as a reduced two-body problem, it is inaccurate for spiral galaxies and has caused great confusion in RC studies. In contrast, the numerical model of Feng and Gallo [3], which summed forces under certain density distributions, showed that gravitational forces in a disk are non-central. Using an analytical approach, Hofmeister et al. [25] proved that the force field around oblate spheroids is likewise non-central. Although gravitational potentials and forces associated with the oblate were historically examined by Gauss and Dirichlet, these early representations are complicated [26], and so the non-central nature of the forces was overlooked in modern studies. In addition, the existence of a center of mass does not require that the mass is concentrated at the center, as exemplified by a bicycle wheel. Consequently, the central point need not be the locus of attraction. Such complications arise whenever the mass distribution is not spherically symmetric, even though Newton's inverse square law is exact for the force between any two mass elements in a body of arbitrary shape.

Early force models presumed that the forces are described by Equation (1) in fitting RC curves [27]. To obtain a good fit required a large, spherical distribution of matter around the flat galaxies: this is the basis of circumgalactic dark matter.

Recent force models do not use Equation (1) but rather sum forces between elements. Specifically, Feng and Gallo [3] computed numerical sums of forces between each pair of mass elements in huge, distributed arrays. Similarly, Marr [4] computed forces directed towards the center by integrating over rings. These force calculations and similar efforts by Pavlovich et al. [5] assumed certain density distributions in order to fit the RC data. Importantly, these essentially correct, but quite different approaches show that dark matter is not needed to fit RC data under various density distributions. However, all such efforts are computationally heavy, forward models.

\subsection{Potential Models and Problems in Applying Poisson's Equation}

Almost all recent orbital models use gravitational potentials to simulate RC. Potentials are presumed to be easier to integrate, using Poisson's equation, than are force elements, because potentials are scalars, rather than vectors. Moreover, once the potential function is known, the force field can be determined by differentiation. Nevertheless, like both early and recent force models discussed above, RC models utilizing potentials are also multi-component forward fits.

In detail, NOMs based on potentials presume that galaxies are constituted of multiple components where each has some arbitrary density distribution, so the net assembly is described by a surfeit of free parameters, commonly 4 to 9 (e.g., [28,29]). A model is commonly considered to be "verified" if the simulated RC is similar to some observed one. On the contrary, the fits of NOMs are known to be equivocal (e.g., [28]). Ambiguity is underscored by the arbitrary practice of minimizing the dark matter component (e.g., [29]), and the obvious fact that the number of fitting parameters far exceeds the number of variables.

Hofmeister and Criss [7] discuss several mathematical errors in recent orbital models involving potentials. The most serious problem is the commonly used, but incorrect, modification of Poisson's equation that employs density sums. This approach, after Dehnen and Binney [30], assumes that the solutions to inhomogeneous differential equations can be superimposed, which is contrary to proofs in elementary textbooks (e.g., [31]). Another problem is the common use of logarithmic potentials, which can only describe a line source like a wire, a shape that is unlike any astronomical body. Another class of problems is associated with the gravitational, vertical instability of shapes other than oblate spheroids, which are therefore prone to collapse.

Another major problem with Poisson's equation lies in its implementation. Investigating flat disks by using Poisson's equation is popular (e.g., [18]), but integration of Poisson's equation requires a resultant finite volume. While Poisson's equation correctly reduces to the density inside a minute 
volume, for any finite volume this equation must be consistent with Gauss' law. To properly implement either Poisson's or Gauss' equation, a coordinate system with appropriate symmetry must be used, and the density distribution about that origin must be known for proper integration. If the mass distribution is spherically symmetric or if density is constant, Poisson's equation offers no advantage in RC calculations, and if not, this equation is nearly impossible to integrate. As discussed by Hofmeister and Criss [7] and Halliday and Resnick [32], the theorem of Gauss provides a much better starting point for such problems, as only a single integration is required, rather than two, thereby removing much ambiguity and facilitating visualization.

Finally, forces around oblate spheroids are non-central, which means that the exterior potential is not simply inversely proportional to the radius even in the equatorial plane [25]. The external potential to oblate bodies is a complex function of cylindrical radius, involving square roots and arcsines.

\subsection{Virial Theorem for Spin}

Galaxies closely resemble oblate spheroids, as demonstrated in the contours of radio emissions [33] and in many visual images. Because galaxies are also spinning, bound systems, their energetics can be specified by the Virial theorem of Clausius. Hofmeister and Criss $[6,14]$ utilized the VT to provide a new class of RC models. Prior use of the VT in the RC literature (e.g., [34]) is limited to calculation of orbits based on Equation (1).

The Virial Theorem directly links the gravitational self-potential of a spinning object $\left(U_{g}\right)$ to two times its rotational kinetic energy. For a homogeneous object, the rotational kinetic energy is $I \omega^{2} / 2$, where $I$ is the moment of inertia and $\omega$ is angular velocity. Thus, if rotational energy has not been lost via internal friction, the VT gives:

$$
\omega^{2}=\frac{-U_{g}}{I}
$$

which is valid for any bound, conservative, homogeneous object [14]. The necessary quantities can all be defined for oblate spheroids, a shape required for any spinning, gravitationally bound object. The morphological descriptors of oblate spheroids are the major radius $A$, minor radius $C$, and eccentricity $e$, which are related by:

$$
e=\sqrt{1-C^{2} / A^{2}}
$$

Also well known are the mass $M$, moment of inertia $I$, and the gravitational self-potential of an oblate spheroid spinning about its minor axis. Specifically, for a homogeneous object:

$$
M=\frac{4}{3} \pi \rho A^{2} C \quad I=\frac{2}{5} M A^{2} \quad U_{g}=-\frac{3}{5} \frac{\operatorname{ArcSin}[e]}{e} \frac{G M^{2}}{A}
$$

Note that $U_{g}$ represents the depth of the potential energy well of the object relative to dispersed matter; the equation for this quantity was allegedly derived by Maclaurin, according to [11]. We take this opportunity to point out that Hofmeister and Criss [6] correctly depict $U_{g}$, but Equation (22) of [7] should be corrected by replacing their $a$ with the radius of the sphere of equivalent volume.

For a spinning, homogenous, oblate spheroid, the angular velocity can be determined from the $U_{g} / I$ ratio per Equation (2):

$$
\omega^{2}=\frac{3}{2} \frac{G M}{A^{3}}\left\{\frac{\operatorname{ArcSin}[e]}{e}\right\}
$$

which can be rewritten as:

$$
\omega^{2}=2 \pi \rho G\left\{\sqrt{1-e^{2}} \operatorname{ArcSin}[e] / e\right\}
$$

The term in brackets is a dimensionless geometric factor that is a constant for any particular spheroid, and is unity for a sphere $(e=0)$. Thus, any homogenous spheroid, including all its internal "shells", rotates coherently, which is true whether the object is solid or not. 
For any specified density function, the mass, self-potential, and moment of inertia of the object can all be determined by appropriate integrations. Thus, in the rigid body approximation, the average angular velocity of the object can be determined by Equation (2). Using this approach and assuming a polytropic density distribution, Hofmeister and Criss [7] demonstrated that galactic mass inferred from the rotational velocities at the visual edge is consistent with the mass estimated from luminosity. Specifically, they found that:

$$
M_{\text {in }}=v_{\text {tangent }}^{2} \frac{2}{3}\left(\frac{5-j}{5}\right)^{3} \frac{r}{G} \frac{e}{\operatorname{ArcSin}[e]}
$$

where $j$ is the polytropic index. We improve our computational methodology below.

\section{Application of the Virial Theorem to Inhomogeneous, Oblate Objects}

\subsection{Dependence of the Spin Rate on Internal Densification}

To calculate RC for oblate objects with variable density, an equation that can be applied to each internal homeoid is needed, because each homeoid will have a particular angular velocity. A very convenient and new finding that is consistent with Newton's homeoid theorem can be established from the above equations by differentiation. This procedure shows that the rotation rate $\omega$ of each independent homeoid within a spheroid can be calculated from the VT. The result is analogous to Equation (5), except that the total mass $M$ and major radius $A$ of the spheroid must be replaced by $M_{\text {in }}$ and $a$, representing the mass interior to each homeoid of major radius $a$. Similarly, Equation (6) will apply to any given homeoid if the density is replaced by the average density $\rho_{\text {in }}$ of matter interior to its major radius. Consequently, recasting Equation (5) in terms of the equatorial tangential velocity gives:

$$
v^{2}=\frac{3}{2} \frac{G M_{i n}}{a}\{\operatorname{ArcSin}[e] / e\}
$$

where $M_{\text {in }}$ is:

$$
M_{\text {in }}=\int_{0}^{a} 4 \pi r^{2} \sqrt{1-e^{2}} \rho_{r} d r
$$

The latter equations allow the equatorial tangential velocity $(v=\omega r)$ to be directly calculated as a function of radial distance $a$, for any assumed density distribution in a spheroidal object of eccentricity $e$. This constitutes forward modeling, which is the standard approach to RC. Figure 1 shows calculations for two different density distributions, which are utilized in diverse problems of astronomical interest, power laws (e.g., [35]) and the venerable polytrope [36,37]. 

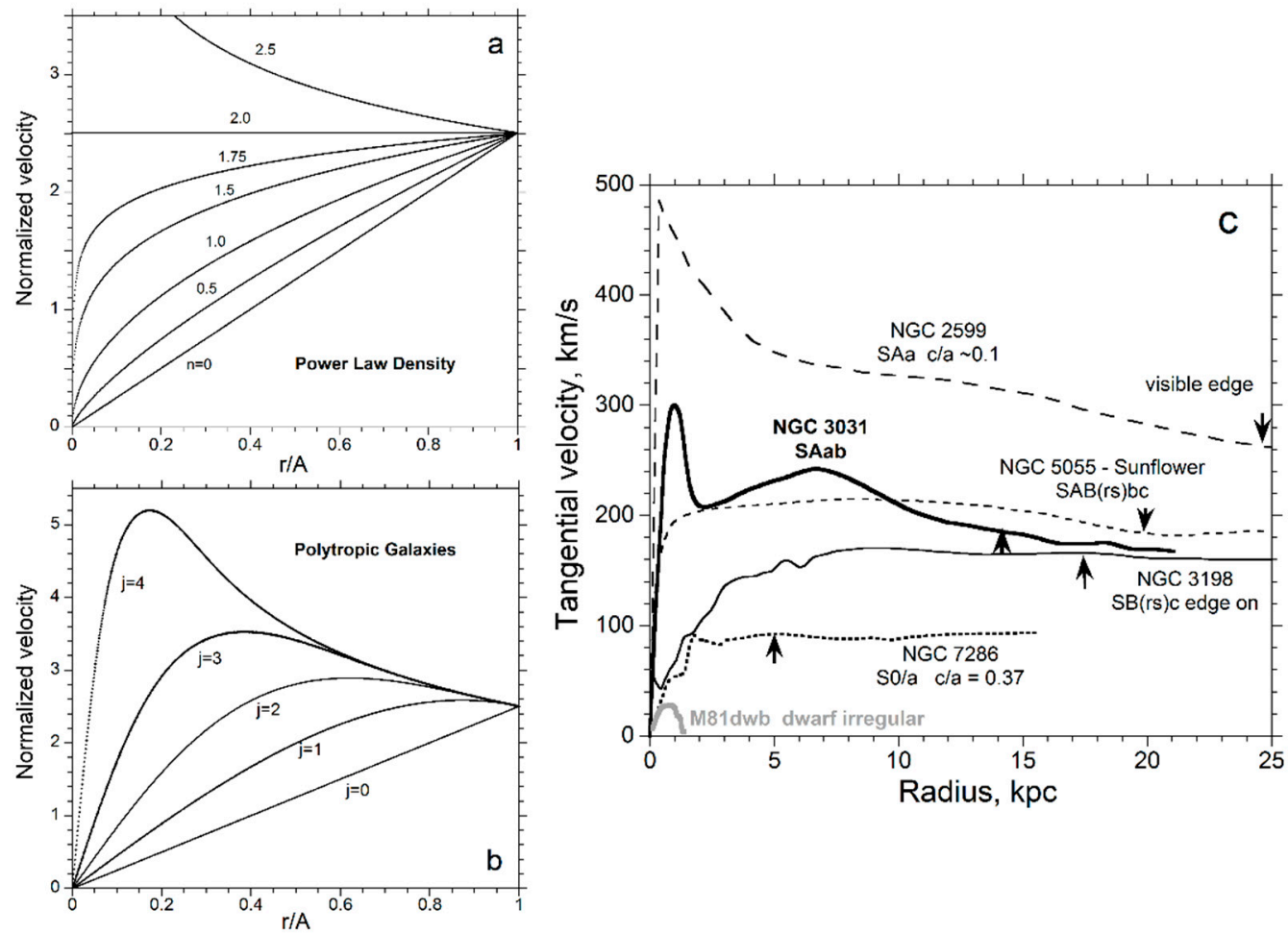

Figure 1. Forward models of galactic rotation curves presuming (a) a power law density distribution for various powers (Equation (15)), and (b) a polytropic density profile of indicated index, using the tabulated density coefficients of [38]. All bodies are presumed to have the same normalized mass of $4 \pi / 3$ at $r=A$, representing unit mean density. Vertical scale is for a spherical object; curves for any other spheroid will have identical shapes but the vertical scale will differ by a geometric factor. Please note that the $n=0$ power law and the curve for polytropic index $j=0$ both represent homogeneous density, in which case the angular velocity is constant throughout the object, requiring that tangential velocity increases linearly with radius. (c) Measured rotation curves for selected galaxies; the data source for M81dwb is [39]; NCG 2559 and 7286 data are from [40], whereas the remainder are from [41].

\subsection{Inverse Model of Galactic Mass}

Most importantly, the inverse approach can be used to directly calculate the galactic mass interior to any equatorial radius of interest, using only the observed equatorial velocity at that distance (Equation (8)). No free parameters and no specific density function are needed. Figure 2 applies this approach to the same set of data on 356 galaxies used by [7], which represents virtually all galaxies for which both RC data and luminosity measurements are reported in the NED database [42]. The calculated masses in Figure 2 tend to be about $3 \times$ larger than those expected if the galactic luminosity is proportional to Solar luminosity in exactly the same proportion as galactic mass is to solar mass. The small $3 \times$ discrepancy is consistent with the latter assumption not being strictly accurate. Different proportionality constants hold for mass and luminosity for three reasons: (1) galaxies contain much gas and dust that is not luminous in the visible; (2) some light is attenuated by the considerable mass external to the visible edge of each galaxy (see Figure 3, below); and (3) light from distant galaxies is attenuated by scattering and absorption by our own Milky Way.

Also relevant are uncertainties in luminosity and velocity measurements. Uncertainty in luminosity is commonly $50 \%$ according to NED, but values are even less accurate for small galaxies. $\mathrm{RC}$ are best known for the large spirals, but velocity dispersion curves used for the smaller dwarfs and ellipticals have considerably greater uncertainties because the patterns are not regular, as in spirals (see e.g., the figures in [43]). 


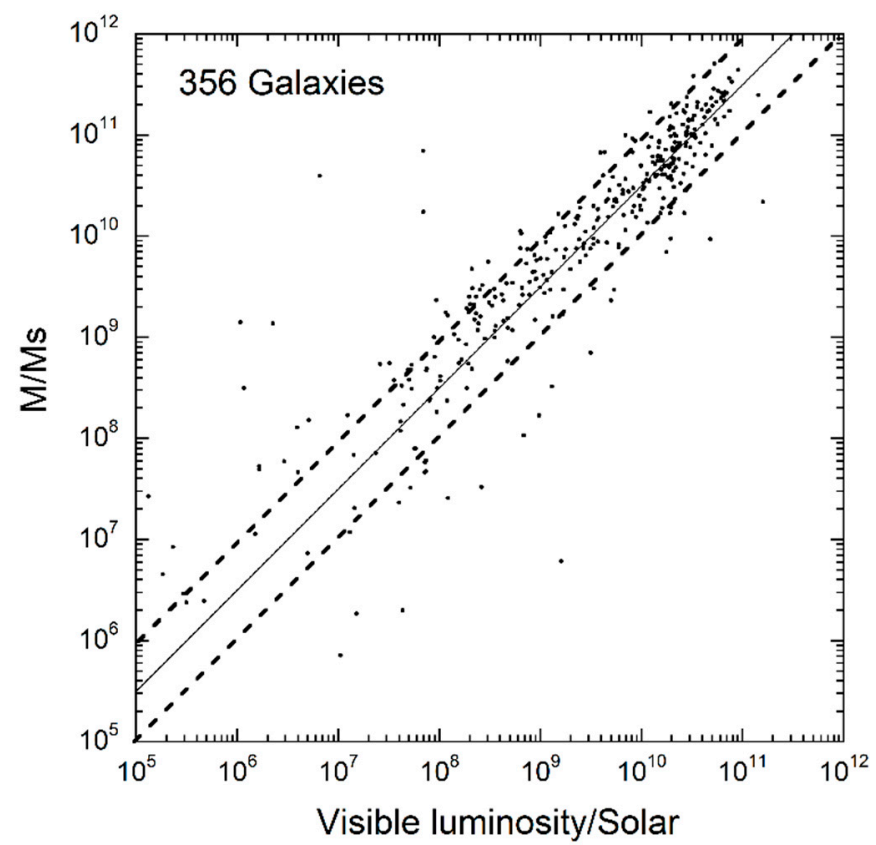

Figure 2. Direct determination of galactic mass interior to the visual edge, using Equation (8) and the 356-galaxy data set of [7]. The calculated mass $M$ and measured visible luminosity of each galaxy are normalized to Solar values. Most calculated masses are about $3 \times$ larger than the normalized luminosity (solid line); the parallel dashed lines are for equal values, and $10 \times$ equal values.

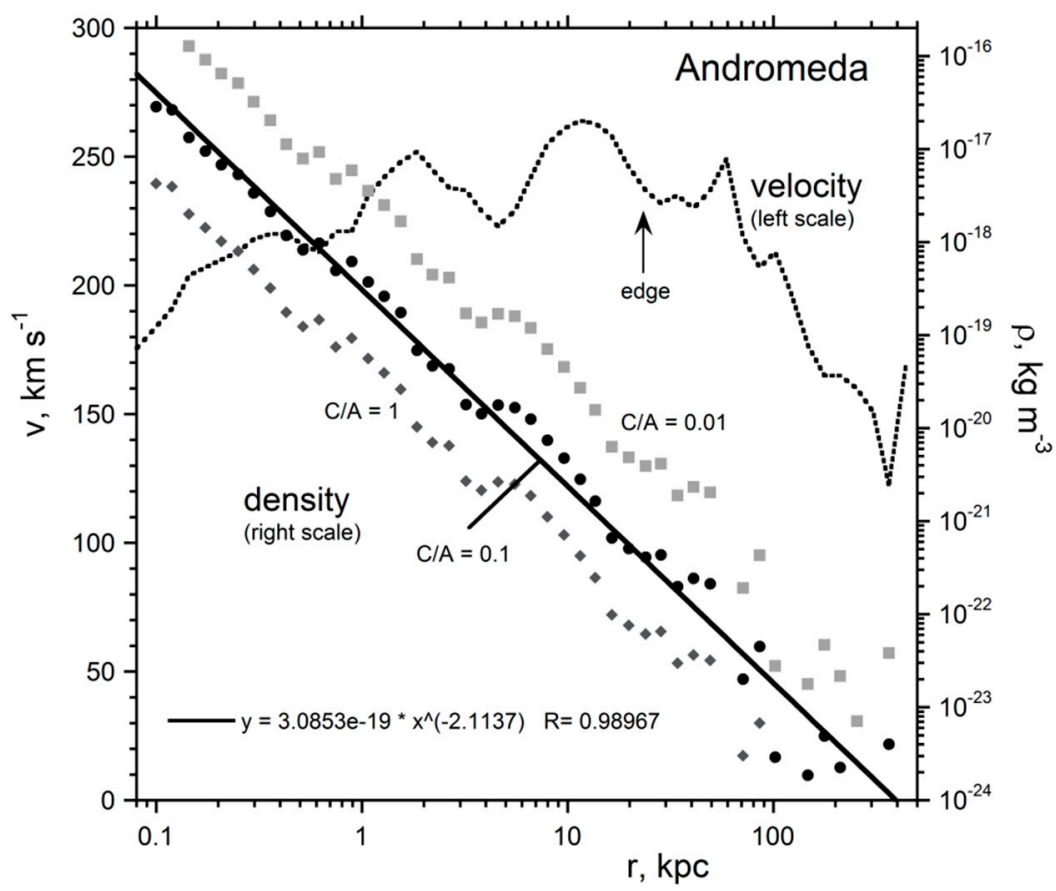

Figure 3. Velocity curve (dotted line, left scale) for Andromeda (RC from [34]), and the corresponding density profiles (right scale) calculated by the inverse model of Equation (10). The effect of shape is illustrated for three choices of the C/A ratio, 1 (diamonds), 0.1 (dots), or 0.01 (squares). Densities in Andromeda span at least 7 orders of magnitude, but vary regularly, approximately conforming to a power law with $\mathrm{n} \sim 2$. Please note that much mass lies beyond the visual edge, whose radial position is shown by the vertical arrow. 


\subsection{Inverse Modeling of Galactic Density}

An important feature of the Virial model is that we were able to mathematically invert it in a manner that allows direct calculation of galactic density profiles from rotation curves. Recasting the above equations in terms of tangential velocity, differentiating, and then rearranging terms yields density as a direct function of velocity and its derivative at any radius in the equatorial plane:

$$
\rho_{a}=\frac{1}{6 \pi G}\left(\frac{2 v}{a} \frac{d v}{d a}+\frac{v^{2}}{a^{2}}\right)\left\{\frac{e}{\sqrt{1-e^{2}} \operatorname{ArcSin}[e]}\right\}
$$

where $a$ is the radial distance in the equatorial plane. For a sphere, the dimensionless geometric factor (in curly brackets) is unity.

This exact, quantitative inverse model allows detailed density profiles to be directly extracted from galactic rotation curves, using only a spreadsheet. Results for the Andromeda galaxy are shown in Figure 3. Criss and Hofmeister [44] calculate similar density profiles for 51 other galaxies, focusing on morphologies common in spirals.

The densities extracted for Andromeda and many other galaxies conform to independent estimates of density in several astronomical environments [44]. For example, near the galactic center, density is like that of molecular cloud cores. At a distance of $8 \mathrm{kpc}$, similar to the distance of the Sun from the rotational center of the Milky Way, the density is similar to estimates of about $10^{-21} \mathrm{~kg} / \mathrm{m}^{3}$ that are available for the interstellar medium [45] and the Solar Neighborhood [46]. Densities obtained where RC terminate are about 3 orders of magnitude lower (e.g., Figure 3), which is consistent with the rarified nature of the intergalactic medium.

Also, note that much of Andromeda's mass resides beyond the visual edge, a finding of relevance to the attenuation of luminosity, mentioned above.

\section{Galactic Evolution}

The comprehensive review of galactic evolution models by Conselice [21] does not discuss interior rotational motions. Instead, modern models focus on external effects such as mergers. Below we construct an evolutionary model of the dynamical behavior of galactic interiors based on the results of Section 3, while considering a few additional physical principles. Radial symmetry is assumed because rotation curves are functions of the cylindrical radius only.

\subsection{Dependence of Evolution on $U_{g}$}

The direction of galactic evolution can be gleaned from the dependence of $U_{g}$ on shape, under the precepts that energy of the system must be minimized and angular momentum conserved. The constraints are as follows. Because the Virial theorem requires that $U_{g}=-2 R E$, and the total energy (TE) is clearly the sum of $U_{g}$ and $R E$, then:

$$
T E=-R E=\frac{U_{g}}{2}
$$

Thus, a process that minimizes the gravitational self-potential will also minimize the total energy of the system. Energy minimization is a hallmark of evolving systems. Based on the well-worn thermodynamic approach of approximating changes as a series of nearly equilibrium steps, we probe the evolution of galaxies in a conservative model. On this basis, and considering galactic morphologies, we deduce that three major steps are involved in galactic evolution. These processes probably overlap in time, but are here separated into a logical sequence that allows for quantitative analysis. 


\subsubsection{Stage 1: Initial Collapse}

Galaxies can be detected only after a significant number of stars have been ignited, so their earliest forms cannot be observed. However, the prevalence of very flat shapes that are not disks, but rather are highly flattened oblates, is suggestive of a vertical collapse. A similar process was proposed as the earliest step in forming the Solar system [47] and was called "pancaking." That paper modeled the formation of the planets subsequent to a vertical collapse but did not provide a quantitative model of the pancaking.

Here we demonstrate that the energy of a spinning molecular cloud can be lowered by vertical collapse that preserves its angular momentum $(L)$, total mass, and internal homogeneity. The mathematics are straightforward. For homogeneous objects, Equation (2) can be recast as:

$$
L=\sqrt{-I U_{g}}
$$

Making appropriate substitutions using Equation (4) provides:

$$
L=\sqrt{\frac{6}{25} G M^{3} A \frac{\operatorname{ArcSin}[e]}{e}}
$$

To describe the flattening, we ratio this result in the angular momentum $L_{o}$ of the homogeneous, spherical body of equal mass and radius $A_{0}$ :

$$
\frac{L}{L_{o}}=\sqrt{\frac{A}{A_{o}} \frac{\operatorname{ArcSin}[e]}{e}}
$$

Simultaneous conservation of angular momentum and mass requires that the term inside the square root must equal unity. This requirement:

$$
\frac{A_{0}}{A}=\frac{\operatorname{ArcSin}(e)}{e}
$$

defines both $U_{g}$ and density of the oblate formed. Figure 4 a shows all three quantities after the collapse as dimensionless ratios.
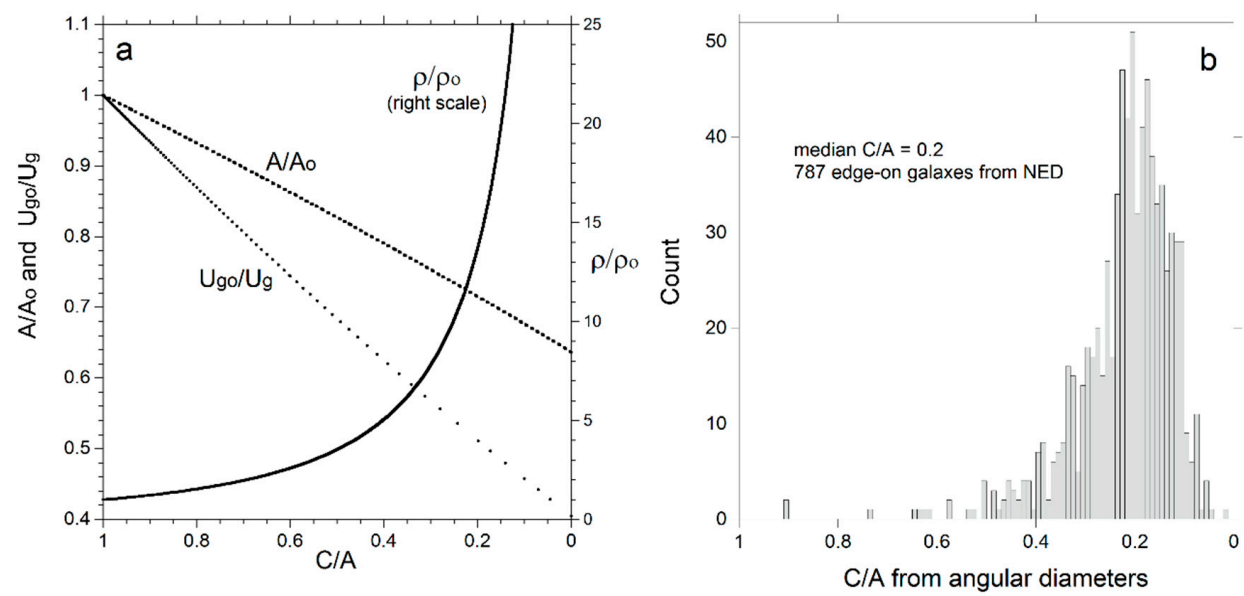

Figure 4. (a) Effect of vertical collapse on the characteristics of spinning oblate clouds. Ratios were obtained from Equation (14b). The trends for $A / A_{o}$ and $U_{g o} / U_{g}$ are nearly, but not precisely, linear on this graph. Please note that the $\mathrm{x}$-axis is reversed, so progress is from left to right, as the nebula evolves into progressively denser, flatter shapes that have lower total energy. (b) Histogram of 787 edge-on galaxies from NED [42]. Note that the high abundance of galaxies at a $c / a$ ratio of 0.2 corresponds to the sharp increase of density at similar $c / a$ ratios in Figure $4 a$. 
Because little contraction in the radial direction occurs, angular momentum about the major spin axis is demonstrably preserved, while net momentum about other axes remains null. The total mass remains constant, but volume decreases as the eccentricity increases, and thus the density increases, while the object remains homogeneous. Thus, an equant, spinning molecular cloud can lower its gravitational potential energy, and hence lower its total energy (Equation (11)), by collapsing into a flatter shape with a somewhat smaller major radius but a significantly higher density, while preserving its angular momentum, total mass, and internal homogeneity, and adhering to the Virial relationship (Figure 4a).

Stars will begin to condense from the molecular cloud when the density increases sufficiently. Thus, this conversion is viewed as an early process in galactic evolution, producing flattened, oblate shapes that are the hallmark of spiral galaxies. This essential first step probably explains the abundance of spiral galaxies.

\subsubsection{Stage 2: Inward Densification}

Central bulges of various sizes are a common, but not ubiquitous feature in spiral galaxies, suggesting that the second stage of galactic evolution involves densification of its central zones. To quantify this process, we assume that galaxy shape and size are preserved.

Any central densification will lower $U_{g}$ of the body, so the total energy continues to be lowered. However, for each homeoid, the angular velocity, moment of inertia, rotational energy, and gravitational energy are proscribed by these constraints, so it is not possible to also simultaneously conserve both the mass and the angular momentum of the total object. Unlike the collapse described above, $L$ and R.E. both change, making it difficult to simultaneously conserve both, since $L=I \omega$ and R.E. $=1 / 2 I \omega^{2}$.

A general relationship that describes contraction does not exist due to tradeoffs. Therefore, specific cases need to be addressed. A power law is used as an example here, because the galactic density of Andromeda (Figure 3) and the other galaxies studied by Criss and Hofmeister [44] approximately follow power laws, except that the trends become flatter in their innermost zones.

We assume that the original body has a homogenous density $\rho_{o}$ and an initial angular momentum of $L_{0}$, but that contraction yields a power law upon densification:

$$
\frac{\rho}{\rho_{o}}=\frac{3-n}{n}\left(\frac{A}{r}\right)^{n}
$$

Conserving shape, volume, and mass yields:

$$
\frac{L}{L_{o}}=\frac{30}{9} \frac{3-n}{10-3 n}
$$

For example, for the $n=2$ case, $1 / 6^{\text {th }}$ of the original inventory of angular momentum must be lost during the densification of the object's central zones. Yet, as is well known, angular momentum must somehow be conserved!

While the vast timescales of galactic processes challenge human imagination, hurricanes are spinning, gaseous objects that can be observed as they evolve, and thereby provide important analogues as to how angular momentum can be conserved as galactic densification proceeds. Careful examination of satellite images shows that, as the inner parts of hurricanes spin up and move radially inward, the outermost cloud belts actually move outward (e.g., https:/ / www.weather.gov/ilm/HurricaneFran; https: / / www.youtube.com/watch?v=6acYq5WgtLU). A series of snapshots of Hurricane Ike (Figure 5) shows the essential changes. As mass is lost and hurricane energy dissipates, the peak velocity decreases markedly. Similarly, maximum velocity of spiral galaxies depends on size and thus on mass, as shown in Figure 1c, and as indicated by Equation (8), which was also confirmed for the 356 galaxies of Figure 2. 
An analogous process appears to occur in spiral galaxies, where the outermost parts of the spiral arms move radially away as the galactic interior densifies. Evidence exists among the subgroups of the SA galaxy class (Figure 6), which shows that spirals with no discernable arms (type 0/a) are only slightly smaller than those with very tight arms (types a and ab). As the arms become progressively more distinct, i.e., better separated from the core or bulge (types a to bc), the average size of SA galaxies remains the same, at about $42 \mathrm{kpc}$. A logical interpretation is that the central zones have densified as the arms have spread out.
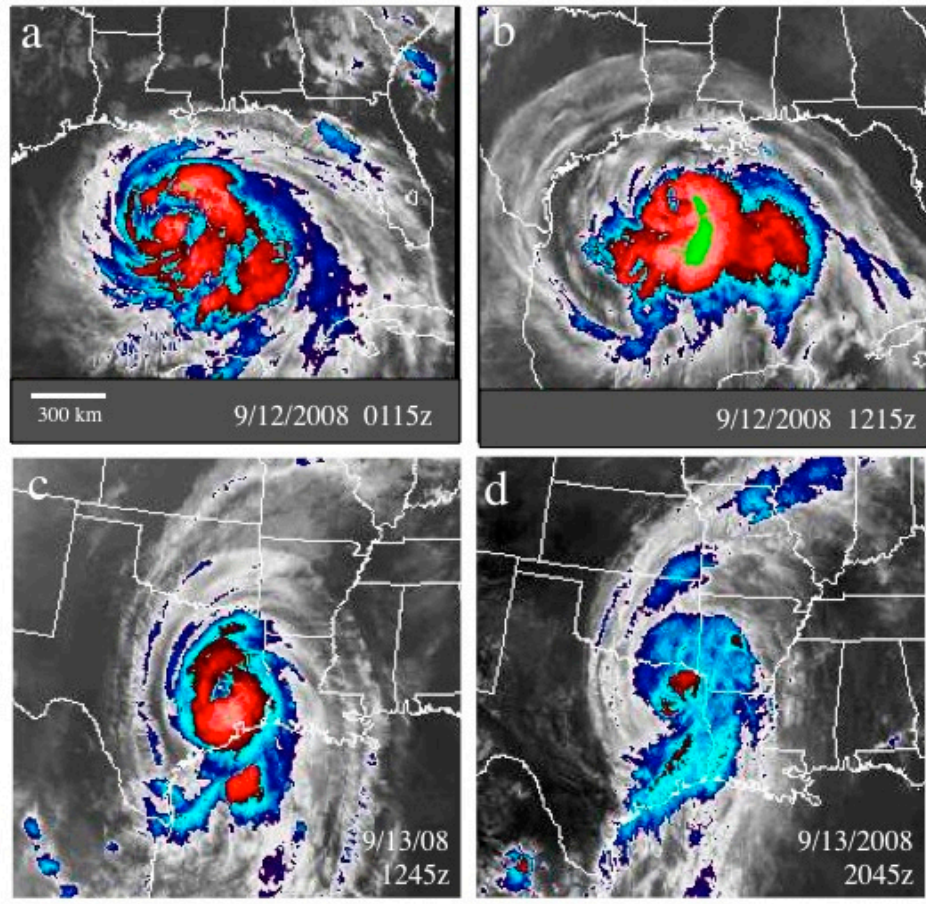

Figure 5. Evolution of Hurricane Ike, a destructive hurricane that rapidly lost strength after making landfall near Houston TX at Category 2 strength. Note how the cloud belts expanded and lost tightness as hurricane strength dissipated from panel a to $\mathrm{d}$, with date and time as listed. A morphologically similar evolutionary sequence probably occurs in spiral galaxies. Images are publicly available from NOAA [48], all at the same scale.

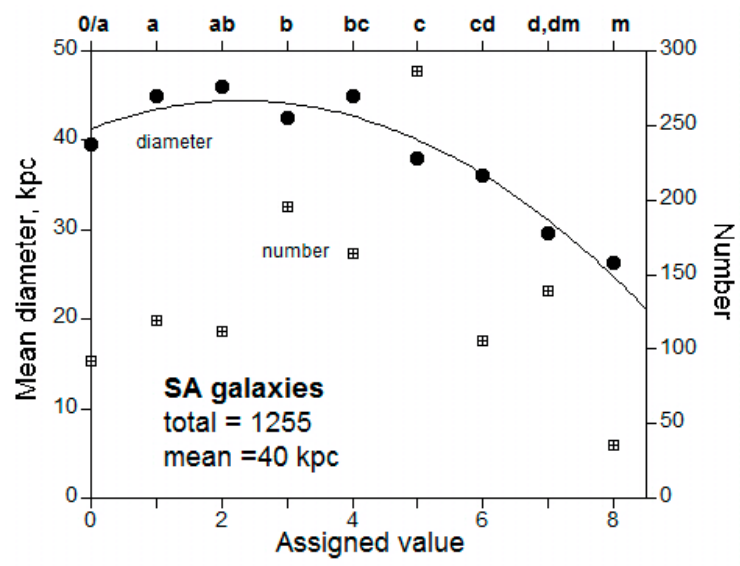

Figure 6. Dependence of the mean diameter on arm type for SA galaxies. Type SA0/a is shown at the origin. Squares indicate the number of galaxies in each category (upper x-axis) in the NED database [42], which provided the necessary data for 1255 SA galaxies. The lower $x$-axis shows integer values assigned to each type for facilitated plotting. On this diagram, galactic evolution progresses left to right. 
Initially, mass can be conserved by redistribution and organization. However, continued outward movement of the spiral arms moving will promote mass loss, which brings us to the next stage of evolution.

\subsubsection{Stage 3: Continuing Densification and Round-Up}

The final, protracted stage of galactic evolution involves continued densification and round-up. This possibility is suggested by Equation (4), which shows that the $U_{g}$ of an object of given shape will decrease, i.e., become a larger negative number, if its major radius decreases, which will increase density.

The effect of shape can be inferred by comparing $U_{g}$ (Equation (4)) for two oblates with differing eccentricity, but with identical mass and volume:

$$
\frac{U_{g 2}}{U_{g 1}}=\left(\frac{e_{1}}{e_{2}}\right)\left(\frac{\operatorname{ArcSin}\left[e_{2}\right]}{\operatorname{ArcSin}\left[e_{1}\right]}\right)\left(\frac{1-e_{2}^{2}}{1-e_{1}^{2}}\right)^{1 / 6}
$$

According to Equation (17), $U_{g}$ of an object will decrease if it becomes more spherical, if the mass, volume and density remain constant. Thus, both densification and roundup will lead to minimization of $U_{g}$ and the total energy, so are consistent with continued evolution in this direction. The effect of simple roundup on lowering of $U_{g}$ is shown in Figure 7.

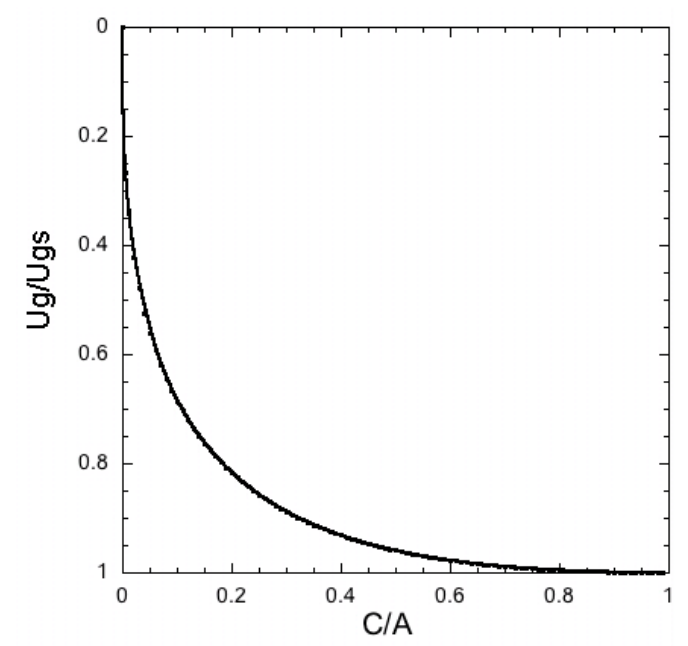

Figure 7. Ratio of the gravitational potential energy of a homogeneous, oblate object $\left(U_{g}\right)$ with aspect ratio $\mathrm{C} / \mathrm{A}$ to that of a homogeneous sphere $\left(U_{g s}\right)$ with the same density, mass and volume, calculated with Equation (17). Because $U_{g s}$ is negative, energy is lost as objects become progressively "rounder", and for this reason the vertical axis is reversed.

Figure 6 provides evidence that mass loss accompanies the continued outward movement of the spiral arms. Because the arms are moving away, the edges of the spirals are dissipating. Types with fairly open arms (c) tend to be smaller than those with tight arms (a to bc), because galaxy size is gauged by luminosity and any isophote requires a certain density of stars. As the arms become more rarified (type cd), the galaxy body becomes smaller, and is the smallest for those lacking arms ( $\mathrm{d}$ to $\mathrm{m}$ ). The regular trend in Figure 6 suggests that as the arms move outward (from bc to m), net mass is lost from the galaxy to space, to offset the increase in angular velocity of the remainder. In this manner, all conservation laws can be satisfied, but they require loss of mass and angular momentum from the galaxy itself to the surroundings during densification. If indeed the Sd and Sm-type galaxies are dissipating, perhaps the end product of spiral evolution is a small irregular or dwarf spheroidal galaxy as a remnant core. 
Additional evidence that roundup is accompanied by mass loss is evident in edge-on spirals (Figure 8). Small spirals are both rounder and have a wider range in C/A than large galaxies. Moreover, Criss and Hofmeister [44] show that large galaxies have denser cores than small ones.

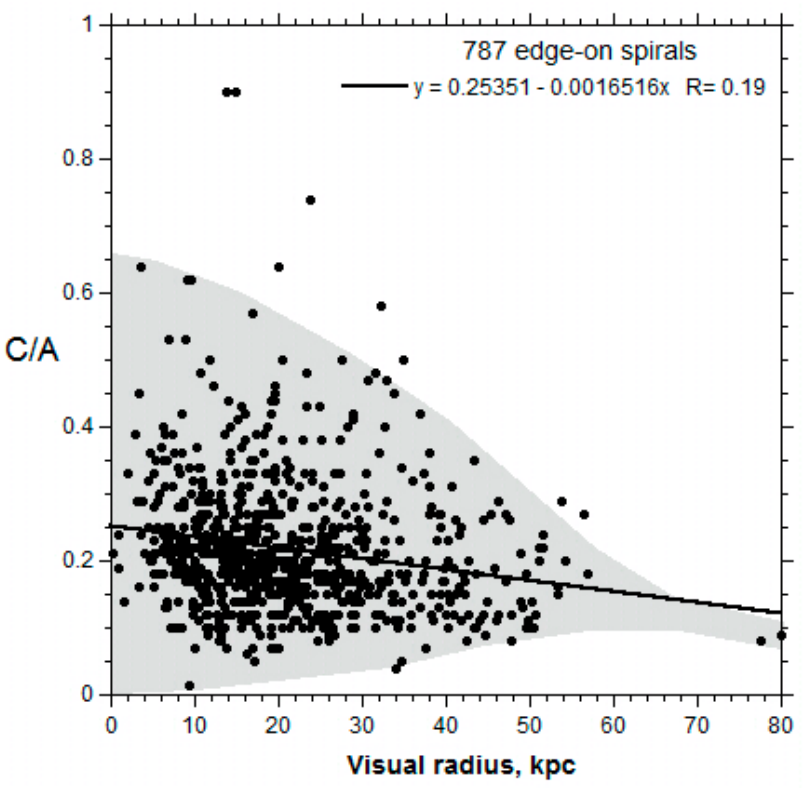

Figure 8. Dependence of the axial ratio on radius for galaxies categorized as edge-on in the NED database [42]. The linear least squares fit excludes one spiral with $A=235 \mathrm{kpc}$ and $C / A=0.061$. The grey area shows that the spread in $C / A$ ratios decreases with galaxy size. Our analysis shows that galactic evolution progresses right to left on this diagram.

\subsubsection{Timing}

Some overlap in time is likely for the three aforementioned stages. However, why should the evolutionary process of flattening (stage 1) be followed by conservative roundup (stage 2), and then by non-conservative roundup (stage 3 )? In all processes, $U_{\mathrm{g}}$ is progressively minimized. However, vertical collapse must occur first for the flat spiral shapes, to produce their defining characteristic. Moreover, the roundup processes require a flat shape as the starting point. So then the question becomes, why does stage 2 precede stage 3, as suggested by Figures 6 and 8 ? The solution to this riddle is that dissipative processes such as diffusion of heat and mass require time, whereas conservative processes, such as phase transitions, can occur rapidly. Hence, stage 3 processes should be the slowest because these do not conserve angular momentum and mass, in contrast to stage 2 or 1 . Over time, significant energy and momentum are lost during radiation to space as well as by inelastic collisions and other interactions. In a non-conservative system, the virial equation no longer defines the spin rate, but because energy is progressively minimized, old, round, internally densified objects such as small or dwarf E0 galaxies will eventually form. An analogue on a much smaller scale are the globular clusters, which likewise are nearly spherical, internally densified, and composed of very old stars.

Of course, the simple picture presented here is that of an average spiral galaxy. We have not addressed the many and varied types of galaxies, but have focused on the commonalities of the spiral class, especially the most symmetric SA type. Over the long time scales involved, interactions will occur between nearby galaxies, and some galaxies will collide with others, producing varied destabilized forms.

\subsection{Galaxy Types that Are Not Flat}

We have shown above that a giant molecular cloud that is spinning will collapse, and suggested the evolutionary behavior of the resulting spiral types. Two other types of galaxies exist; denoted as 
lenticulars and ellipticals. These galaxies are "round," unlike spirals, and tend to be much larger than spirals (Figure 9). In fact, ellipticals are categorized by their roundedness.
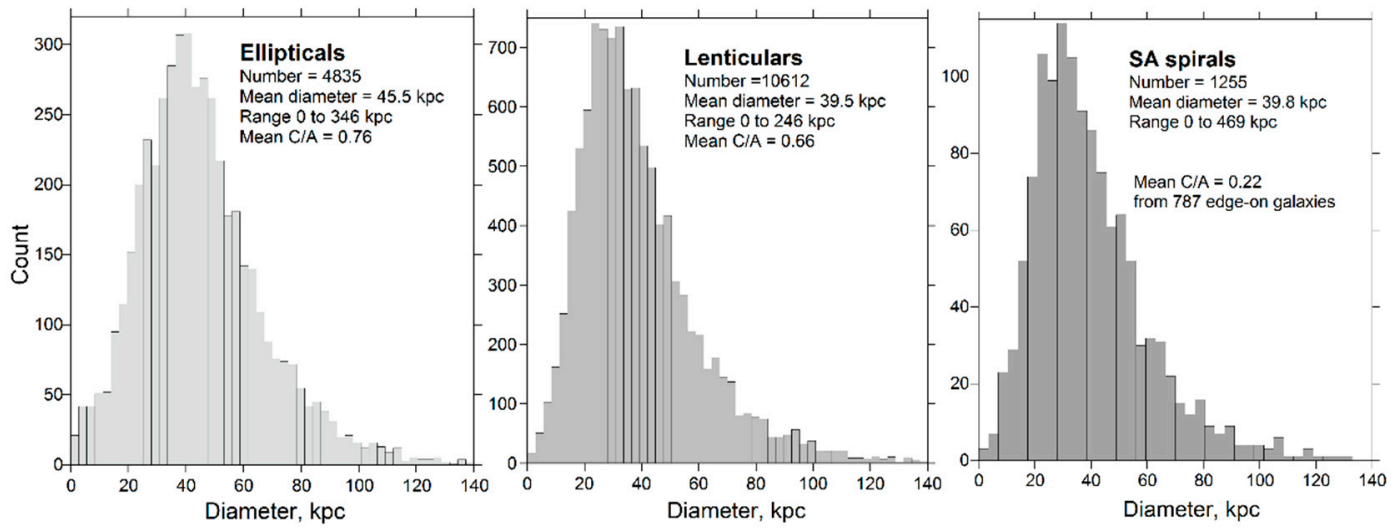

Figure 9. Histograms of important galaxy types. Data were mined from NED [42] in the stated groupings. The elliptical category was trimmed of all types except those labeled E, which mostly removed very small galaxies, although the mean was little affected. The lenticular download was cleaned of types that are likely spirals, such as SA0/a, which are represented in the SA group. For each of the ellipticals and lenticulars, C/A was determined from a smaller subset, and for the spirals the edge-on category of NED [42] was used. Due to being flat, the spirals with the same A as the ellipticals have much lower volume. The similar shapes of these skewed histograms reveals that similar processes are common to all, see Apostle [49] for a discussion of the link of mathematics of histograms to the underlying physics.

The third very important difference regards the nature of galactic rotation. Elliptical galaxies do not exhibit coherent rotation; instead, their internal motions are described as random orbits about a center and the velocity measurements are described as "dispersions". For graphical representations of the 3-d motions, see [43]. The velocity dispersions can be "collapsed" into a function of radius, and then resemble the RC (e.g., [50]), even though coherent rotation does not exist. This behavior is irrespective of the size of the elliptical. Early type disk galaxies were studied by Noordemeer et al. [40]. Of the 68 galaxies in their survey, only 19 were found suitable for their analysis, and the most lenticular of the objects studied, one each of SA0-, SA0/a, SA(r)0+and SA(r)0/a, are often grouped as spirals (note that NGC 3993 is no longer classified as an S0). The authors note that some of the data had distorted kinetics. Thus, only spiral galaxies truly have RC. Lenticulars probably have velocity dispersions, given the difficulty in distinguishing type $\mathrm{S} 0$ into the elliptical types, and the difficulty that Noordemeer et al. [40] had in extracting high quality RC from spiral types that grade into lenticular types.

Given the above, the remainder of the present section groups lenticulars with ellipticals. The essential distinction is whether a particular lenticular has coherent rotation, or not. It appears that the classes Sa, Sab and other early type spirals have RC. These should evolve like the SA types described above.

The remainder of this section explores possible evolutionary paths of the round galaxies (ellipticals and S0 types), which are not coherently spinning. Conservation of angular momentum indicates that these types never had significant net angular momentum. Although their shapes need not be oblate because the galaxies are not spinning, their shapes are similar enough to the oblate, so we use this term and the equations above describing their energies. Actually, their internal homeoids are probably spinning, but the axes are random, and collisions are rare due to the distances between stars. 


\subsubsection{No Vertical Collapse}

If a large cloud lacks the coherent motions of spin, it will not undergo a vertical collapse. However, for such a cloud to be stable, the matter must be orbiting randomly about the center, perhaps as shells. Contraction is expected, as are changes in shape. As discussed above, the draw is to a point. However, as also mentioned above, it is difficult to meet all conservative requirements during densification without some material moving outwards.

\subsubsection{Density Redistribution}

Stage 2 of the evolution of spirals was modelled as conservative. Gravity being a central force will draw matter to the center. This requires other material to go out. Although our model for stage 2 (Section 4.1.2) considered the size to not change in order to provide a mathematical formulation, it is also possible for the outside (a mantle) to expand while the inside (a core contracts). Figure 9 provides support, in that the lenticulars, which include the E-S0 class, are, on average, smaller than the ellipticals.

\subsubsection{Rounding Up}

Stage 3 of spiral evolution was modelled as non-conservative, where mass was lost at the edges as the arms disappeared, while the interior became denser and rounder. Ellipticals do not have arms, but an analogous process exists where the outer layers diffuse away. As this material is progressively lost, the evolving objects become smaller and rounder. Supporting evidence exists in data on the ellipticals (Figure 10). Although the trend of C/A vs. 2A is weak, due to the large number of galaxies in the NED database [42], it is clear that such a trend exists.

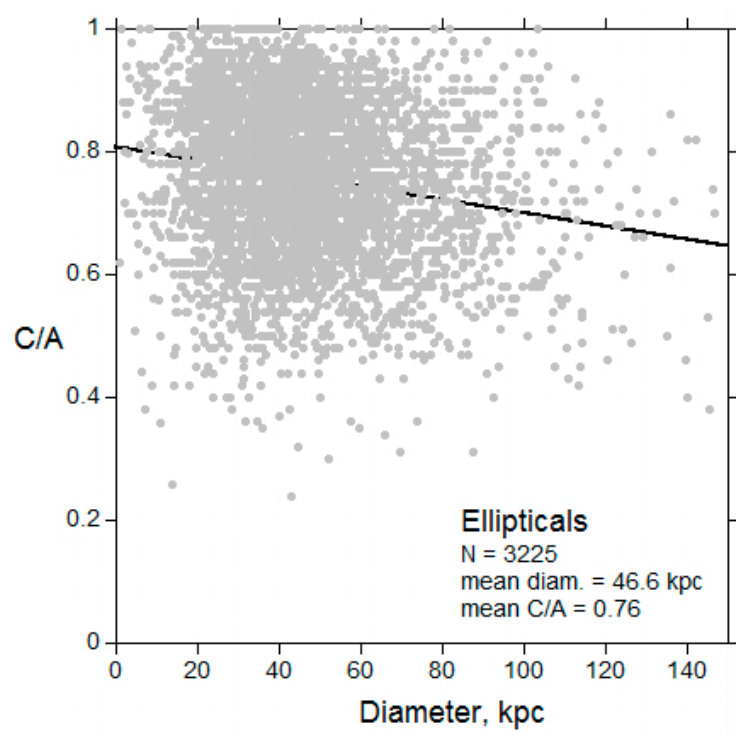

Figure 10. Dependence of the axial ratio on ellipticals in the NED database [42]. The number of samples is lower than that in Figure 8 because all types that were not labeled as E, E1, etc. are excluded. Please note that the mean values were unchanged during the culling.

\subsubsection{Jets}

Another way to achieve round up while conserving angular momentum and rotational energy is for the object to spit out axial jets, which carry away kinetic energy but not angular momentum. Here we assume that the fairly round galaxies have some small net R.E. Being oblates, the forces are non-central [25]. 
To model this phenomenon, we conserve total energy:

$$
U_{g, \text { oblate }}+R E_{\text {oblate }}+U_{g, j e t s}+L E_{j e t s}=U_{g, \text { sphere }}+R E_{\text {sphere }}
$$

where $L E$ is the linear kinetic energy of the jets. To provide an approximate solution, we consider a point distant from the oblate galaxy and its jets: together these objects act as a point mass, providing:

$$
U_{g, \text { oblate }}+U_{g, j e t s} \cong U_{g, \text { sphere }}
$$

Equation (19) is most accurate at the time of jet formation. Combining Equations (18) and (19) gives:

$$
R E_{\text {oblate }}+L E_{\text {jets }} \cong R E_{\text {sphere }}
$$

Equation (20) shows that kinetic energy can be conserved during round up, because the reduction in R.E. is compensated by the kinetic energy of the jets. Total energy is conserved through the Virial theorem and the approximate relationship of Equation (19). Lastly, angular momentum can be conserved by requiring that changes in $I \omega$ balance during round up. A detailed analysis, including mass balance, is not performed here because data on elliptical masses are needed. We simply state here that smaller ellipticals rotate slower (compare data in [43] to that in [50]).

In support of our deduction, many galaxies have radio jets, indicating motion of gas along the vertical axes. These are disproportionately observed in ellipticals. Of the 7266 galaxies in the elliptical category of NED [42], 72 have radio jets. Few of the numerous lenticulars $(12,551)$ have radio jets (46) while the even larger number of spirals $(38,660)$ have only 25 jets. A miniscule number of irregular galaxies have radio jets (2 out of 2711).

\section{Discussion}

Although Hubble carefully avoided over interpreting his famous "tuning fork" diagram in terms of a definite evolutionary sequence, the continuity of forms has some likely relationship to galactic evolution. When combined with requirements for energy minimization and angular momentum conservation, our equations provide useful guides and allow us to suggest a simplified overview of this complex process, summarized in Figure 11. Spiral galaxies must initially form by a rather rapid vertical collapse of a massive, spinning molecular cloud. In this manner, angular momentum can be conserved, densification can occur, and energy can be lowered, and a shape consistent with the Virial theorem and with the shape of a Maclaurin spheroid can be produced. In contrast, simple radial collapse would produce high densities that would require rotation rates inconsistent with a more spherical shape. The apparent necessity of vertical collapse as a first step explains the abundance of spiral galaxies and the occurrence of this shape on the much smaller scale of our Solar System.

Additional densification will lower energy further, but the conservation laws cannot all be simultaneously satisfied. A strong possibility is that mass is lost as energy is minimized, apparently required to conserve angular momentum. We deduce that, as the central part of the galaxy becomes more dense and spins up, the distal parts of the spiral arms move outward, carrying away both mass and angular momentum. An analogous process can be observed during the dissipation of most hurricanes (e.g., Figure 5).

In contrast, elliptical and lenticular galaxies appear to be systematically larger that spiral galaxies, but they have much lower net angular momentum. Thus, they do not undergo an early vertical collapse. Instead, their evolution involves progressive densification, contraction, and round up. When angular momentum cannot be conserved, vertical jets are emitted from their central zones.

Thus, our results suggest that the evolution of any given galaxy depends critically on its initial mass and the magnitude of coherent spin. Large galaxies have more mass to lose than small, and importantly, the weight of a large galaxy is conducive to compressing the interior, as is observed for planets. The large initial mass thus should create a denser and more coherent interior. 
Eventually, galaxies must round up. Major energy loss is required to achieve this state, so much time is probably required. The fact that globular clusters are mostly constituted of very old stars is consistent with this evolutionary view.

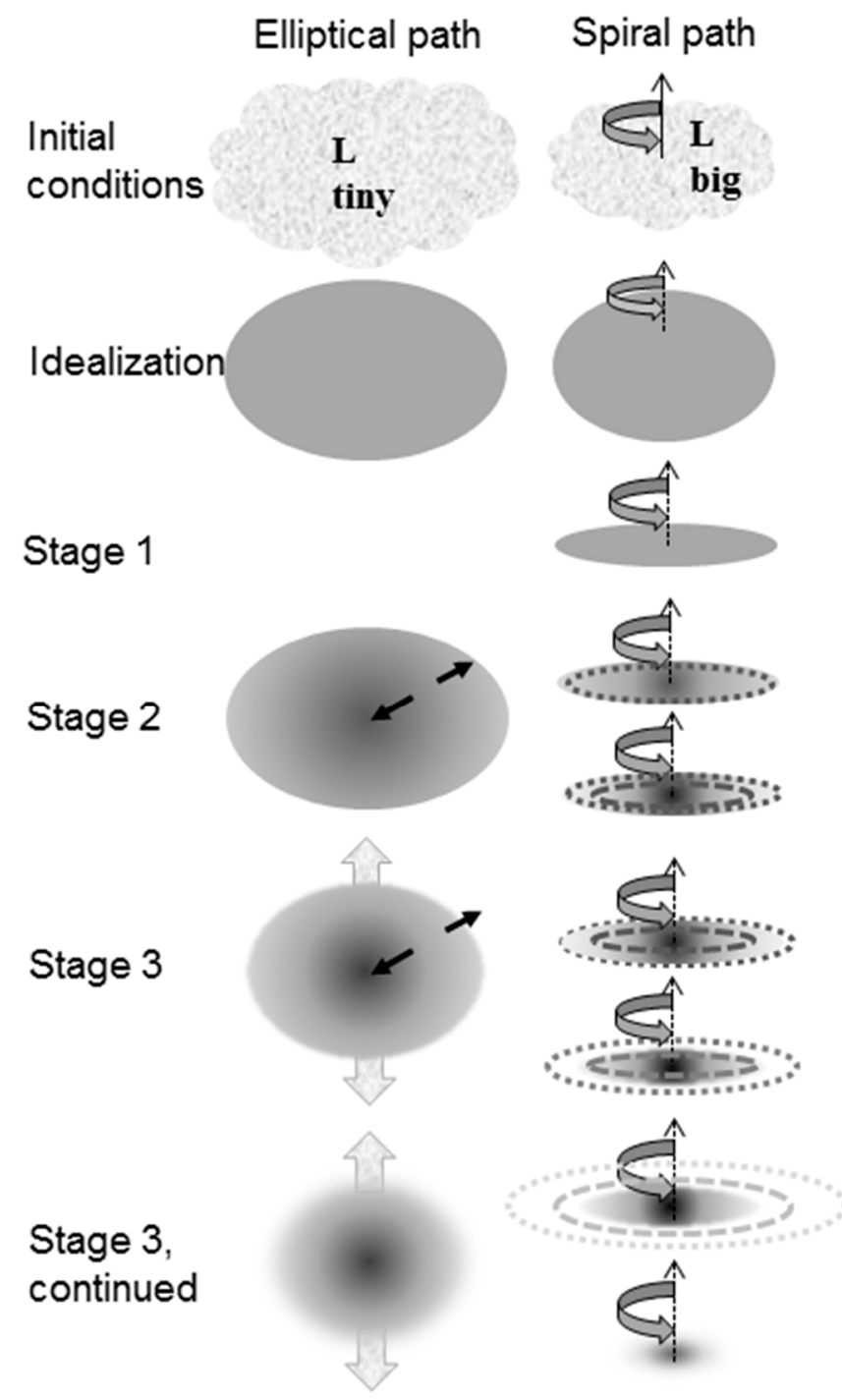

Figure 11. Schematic of largely conservative galactic evolution. Top row = giant precursor clouds. The data suggest clouds for spirals were smaller. Second row $=$ homogenous density oblates assumed to provide analytic solutions. Third row (Stage 1) = pancaking, but only of the coherently spinning clouds. Fourth row (Stage 2) = conservative contraction which densifies the interior and in spirals produces the organized structures, shown here as rings, which also represent the arms. Fifth and sixth rows (Stage 3) = contraction and round up with mass loss. For the spirals, the arms dissipate. For ellipticals, either the outer layers diffuse or jets are produced, which carry away kinetic energy and mass, allowing angular moment and energy to be conserved. Because radial symmetry is presumed, this evolutionary sketch does not show the lower symmetry aspects of bars and arms.

\section{Conclusions}

Our inverse model of galactic rotation curves provides a means to directly calculate volumetric density from the measured rotational velocity and its radial derivative for any oblate object of known eccentricity (Equation (10)). The physical basis is the Virial theorem and Newton's law of gravity, and the mathematics are straightforward and easily verified. Because this is a bona fide inverse 
model, direct calculation of density is made using only observed data. No free parameters, nor other assumptions, fits, scale-lengths, nor approximations are invoked.

We show that density for Andromeda varies by at least 7 orders of magnitude from center to edge, yet decreases according to a simple power law. Most importantly, the values so obtained agree with independent measurements of density in appropriate astronomical environments (ISM and IGM). This comparison validates our model, since the rotation curves of spirals are similarly shaped and the maximum velocities for spiral galaxies occupy a surprisingly narrow range.

Forward modeling using the Virial Theorem explains regularities among galactic rotation curves. This approach can be used to calculate synthetic rotation curves for any presumed density distribution.

Because the internal dynamics of galaxies must influence their evolution, the second half of the paper extends our model to include the influence of time on rotation of oblate bodies. Energy minimization and angular momentum conservation are combined with the Virial theorem to ascertain how rotational velocities can evolve. Spiral galaxies initially form by the vertical collapse of rotating, equant molecular clouds, producing a flattened shape such as an SA0/a galaxy that lowers the self-potential while conserving the object's angular momentum. The self-potential can be further reduced by subsequent, inward densification. However, the conservation of momentum requires mass loss during this process, achieved by the outward movement of the distal parts of the spiral arms. In contrast, elliptical galaxies initially have little net angular momentum, and they evolve into progressively rounder and denser forms, sometimes requiring the development of central, vertically oriented jets.

Our model indicates that galactic evolution is governed by the initial inventory of mass and angular momentum, which result in two diverging paths for elliptical and spiral galaxies that are codified by Hubble's tuning fork diagram. Spiral galaxies have significant angular momentum, and their spinning, internally densified shapes and velocity profiles resemble hurricanes, a similarity that extends to the null central velocities required for spin. Many of the predicted evolutionary patterns of spiral galaxies are exhibited by hurricanes, whose short time and length scales are conducive to human observations.

Author Contributions: Formal analysis, R.E.C.; Validation, A.M.H.

Funding: This research received no external funding.

Acknowledgments: This research has made use of the NASA/IPAC Extragalactic Database (NED), which is operated by the Jet Propulsion Laboratory, California Institute of Technology, under contract with the National Aeronautics and Space Administration.

Conflicts of Interest: The authors declare no conflict of interest.

\section{References}

1. Trimble, V. Existence and nature of dark matter in the universe. Annu. Rev. Astron. Astrophys. 1987, 25, 425-472. [CrossRef]

2. Milgrom, M.A. modification of the Newtonian dynamics as a possible alternative to the hidden mass hypothesis. Astrophys. J. 1983, 270, 365-370. [CrossRef]

3. Feng, J.Q.; Gallo, C.F. Mass distribution in rotating thin-disk galaxies according to Newtonian dynamics. Galaxies 2014, 2, 199-222. [CrossRef]

4. Marr, J.H. Galaxy rotation curves with lognormal density distribution. Mon. Not. R. Astron. Soc. 2015, 448, 3229-3241. [CrossRef]

5. Pavlovich, K.; Pavlovich, A.; Sipols, A. Newtonian explanation of galaxy rotation curves based on distribution of baryonic matter. arXiv 2014, arXiv:1406.2401P.

6. Hofmeister, A.M.; Criss, R.E. The physics of galactic spin. Can. J. Phys. 2017, 95, 156-166. [CrossRef]

7. Hofmeister, A.M.; Criss, R.E. Implications of Geometry and the Theorem of Gauss on Newtonian Gravitational Systems and a Caveat Regarding Poisson's Equation. Galaxies 2017, 5, 89. [CrossRef]

8. Annila, A. Space, time and machines. Int. J. Theor. Math. Phys. 2012, 18, 191-205. [CrossRef] 
9. Todhunter, I. A History of the Mathematical Theories of Attraction and Figure of the Earth; MacMillan and Co.: London, UK, 1873; reprinted by Dover Publications: New York, NY, USA, 1962.

10. Maclaurin, C. Treatise on Fluxions in Two Books; T.W. \& T. Ruddimans: Edinburgh, UK, 1742.

11. Dankova, T.; Rosensteel, G. Triaxial bifurcations of rapidly rotating spheroids. Am. J. Phys. 1998, 66, 1095. [CrossRef]

12. Roberts, P.H.; Stewartson, K. On the stability of a Maclaurin spheroid of small viscosity. Astrophys. J. 1963, 137, 777-791. [CrossRef]

13. Clausius, R. On a mechanical theorem applicable to heat. Philos. Mag. 1870, 40, 122-127. [CrossRef]

14. Hofmeister, A.M.; Criss, R.E. Spatial and symmetry constraints as the basis of the virial theorem and astrophysical implications. Can. J. Phys. 2016, 94, 380-388. [CrossRef]

15. Nicolis, G.; Prigogine, I. Self-Organization in Nonequilibrium Systems: From Dissipative Structures to Order through Fluctuations; J. Wiley and Sons: New York, NY, USA, 1977.

16. Groetsch, C.W. Inverse Problems: Activities for Undergraduates; Cambridge University Press: Cambridge, UK, 1999.

17. Ambarzumian, V. On the derivation of the frequency function of space velocities of the stars from the observed radial velocities. Mon. Not. R. Astron. Soc. 1936, 96, 172-179. [CrossRef]

18. Takamiya, T.; Sofue, Y. Radial distribution of the mass-to-luminosity ratio in spiral galaxies and massive dark cores. Astrophys. J. 2000, 534, 670-683. [CrossRef]

19. Angus, G.W.; Gentile, G.; Famaey, B. Dynamical measurement of the stellar surface density of face-on galaxies. Astron. Astrophys. 2016, 585, A17. [CrossRef]

20. Kurapati, S.; Banerjee, A.; Chengalur, J.N.; Makarov, D.; Borisov, S.; Afansiev, A.; Antipova, A. Mass modelling of a superthin galaxy, FGC 1540. Mon. Not. R. Astron. Soc. 2018, 479, 5686-5695. [CrossRef]

21. Conselice, C.J. The evolution of galaxy structure over cosmic time. Ann. Rev. Astron. Astrophys. 2014, 52, 291-337. [CrossRef]

22. McGaugh, S.S. A tale of two paradigms, the mutual incommensurability of LCDM and MOND. Can. J. Phys. 2015, 93, 250-259. [CrossRef]

23. Lin, H.-N.; Li, M.-H.; Li, X.; Chang, Z. Galaxy rotation curves in the Grumiller's modified gravity. Mon. Not. R. Astron. Soc. 2013, 430, 450-458. [CrossRef]

24. Wiegert, T.; English, J. Kinematic classification of non-interacting spiral galaxies. New Astron. 2014, $26,40-61$. [CrossRef]

25. Hofmeister, A.M.; Criss, R.E.; Criss, E.M. Verified solutions for the gravitational attraction to an oblate spheroid: Implications for planet mass and satellite orbits. Planet. Space Sci. 2018, 152, 68-81. [CrossRef]

26. Schmidt, M. A model of the distribution of mass in the galactic system. Bull. Astron. Inst. Neth. 1956, 13, $15-42$.

27. Sofue, Y.; Rubin, V.C. Rotation curves of spiral galaxies. Ann. Rev. Astron. Astrophys. 2001, 39, 137-174. [CrossRef]

28. Ibata, R.; Lewis, G.F.; Martin, N.F.; Bellazzini, M.; Correnti, M. Does the Sagittarius Stream constrain the Milky Way halo to be triaxial? Astrophys. J. Lett. 2013, 765, L155. [CrossRef]

29. Kam, Z.S.; Carignan, C.; Chemin, L.; Amram, P.; Epinat, B. Kinematics and mass modelling of M33, H $\alpha$ observations. Mon. Not. R. Astron. Soc. 2015, 449, 4048-4070. [CrossRef]

30. Dehnen, W.; Binney, J. Mass models of the Milky Way. Mon. Not. R. Astron. Soc. 1998, 294, 429-438. [CrossRef]

31. Pinsky, M.A. Introduction to Partial Differential Equations with Applications; McGraw-Hill: New York, NY, USA, 1984.

32. Halliday, D.; Resnick, R. Physics; John Wiley and Sons: New York, NY, USA, 1966.

33. Wiegert, T.; Irwin, J.; Miskolczi, A.; Schmidt, P.; Carolina Mora, S.; Damas-Segovia, A.; Stein, Y.; English, J.; Rand, R.J.; Santistevan, I. Changes in Radio continuum emission of 35 edge-on galaxies observed with the Karl G. Jansky very large array in d configuration-Data release 1. Astronom. J. 2015, 150, 81. [CrossRef]

34. Sofue, Y. Dark halos of M 31 and the Milky Way. Publ. Astron. Soc. Jpn. 2015, 67, 759-767. [CrossRef]

35. Binney, J.; Tremaine, S. Galactic Dynamics, 2nd ed.; Princeton University Press: Princeton, NJ, USA, 2008.

36. Emden, R. Gaskugeln—Anwendungen de Mechanischen Wärmetheorie; B. G. Teubner: Leipzig, Germany, 1907.

37. Eddington, A.S. The Internal Constitution of the Stars; Dover Publications: New York, NY, USA, 1959. 
38. Criss, R.E.; Hofmeister, A.M. Analytical representations for simple and composite polytropes and their moments of inertia. New Astron. 2015, 36, 26-31. [CrossRef]

39. Oh, S.-H.; de Blok, W.J.G.; Walter, F.; Brinks, E.; Kennicutt, R.C., Jr. High-resolution dark matter density profiles of THINGS dwarf galaxies, correcting for noncircular motions. Astron. J. 2008, 136, 2761-2781. [CrossRef]

40. Noordermeer, E.; van der Hulst, J.M.; Sancisi, R.; Swaters, R.S.; van Albada, T.S. The mass distribution in early-type disc galaxies, declining rotation curves and correlations with optical properties. Mon. Not. R. Astron. Soc. 2007, 376, 513-546. [CrossRef]

41. Sofue, Y.; Tutui, Y.; Honma, M.; Tomita, A.; Takamiya, T.; Koda, J.; Takeda, Y. Central rotation curves of spiral galaxies. Astrophys. J. 1999, 523, 136-146. [CrossRef]

42. NED: NASA/IPAC Extragalactic Database. Available online: https://ned.ipac.caltech.edu/ (accessed on 26 June 2017).

43. Romanowsky, A.J.; Douglas, N.G.; Arnaboldi, M.; Kuijken, K.; Merrifield, M.R.; Napolitano, N.R.; Capaccioli, M.; Freeman, K. A dearth of dark matter in ordinary elliptical galaxies. Science 2003, 301, 1696-1698. [CrossRef] [PubMed]

44. Criss, R.E.; Hofmeister, A.M. Determination of interior galactic density from parameter-free inverse models of rotation curves assuming differential Newtonian spin. Galaxies 2018, in preparation.

45. Ferrière, K. The interstellar environment of our galaxy. Rev. Mod. Phys. 2011, 73, 1031. [CrossRef]

46. LeDrew, G. The real starry sky. J. R. Astron. Soc. Can. 2011, 95, 322-324.

47. Hofmeister, A.M.; Criss, R.E. A thermodynamic model for formation of the Solar System via 3-dimensional collapse of the dusty nebula. Planet. Space Sci. 2012, 62, 111-131. [CrossRef]

48. NOAA. National Oceanic and Atmospheric Administration. Available online: http://www.noaa.gov/ (accessed on 16 September 2008).

49. Apostol, M. Calculus: Multi-Variable Calculus and Linear Algebra, with Applications to Differential Equations and Probability; Xerox College Publishing: Waltham, MA, USA, 1969.

50. Howley, K.M.; Guhathakurta, P.; van der Marel, R.; Geha, M.; Kalirai, J.; Yniguez, B.; Kirby, E.; Cuillandre, J.-C.; Gilbert, K. Internal stellar kinematics of M32 from the SPLASH survey, dark halo constraints. Astrophys. J. 2013, 765, 65. [CrossRef]

(C) 2018 by the authors. Licensee MDPI, Basel, Switzerland. This article is an open access article distributed under the terms and conditions of the Creative Commons Attribution (CC BY) license (http://creativecommons.org/licenses/by/4.0/). 